\title{
COMMISSION 30: RADIAL VELOCITIES
}

\author{
(VITESSES RADIALES)
}

\author{
PRESIDENT: A.A. Tokovinin \\ VICE-PRESIDENT: B. Nordström \\ ORGANIZING COMMITTEE: D. Dravins, H. Levato, T. Mazeh, N. Morell, \\ H. Quintana, M.A. Smith, L. Szabados \& S. Udry
}

\section{Introduction}

Commission 30 has 129 members and consultants in 28 countries who are active in the area of radial velocities (RVs). The work of the Commission covers a wide range of topics all of which concern the application of the Doppler effect to astronomical objects, including galaxies and the interstellar medium as well as stars, and in all wavelength ranges of the electromagnetic spectrum. Although the commission focusses mainly on stellar and galaxy radial velocities, it is noted that stellar rotation, spectroscopic binaries, extrasolar planets, pulsating stars, asteroseismology and turbulence in stellar atmospheres are also included.

The reader is invited to visit the WEB page of Commission 30 at http:/ /www.ctio.noao. edu/science/iauc30/iauc30.html for detailed information on the Commission and its WGs. An extended html version of this Report with active links is available there.

\section{Scientific Highlights, 2000-2002}

\subsection{The Definition of Radial Velocity}

\section{Dravins}

Very high measuring precisions, differential lineshifts measured in the same star, and astrometric determinations of radial motion, have required the concept of "radial velocity" to be more stringently defined, in order to permit comparisons between various measurements on accuracy levels of $\mathrm{m} \mathrm{s}^{-1}$. Relativistic effects, measurements being made inside gravitational fields, and alternative choices of coordinate frames, cause the naive concept of RV being equal to the time derivative of distance, to become ambiguous at accuracy levels around $100 \mathrm{~m} \mathrm{~s}^{-1}$. Following an e-mail discussion in the community during a couple of years, two resolutions for the stringent definition of spectroscopic and astrometric RVs were adopted at the IAU XXIVth General Assembly in Manchester (Rickman 2002).

For precise spectroscopic measurements, the desired result is the barycentric radialvelocity measure, $c z_{\mathrm{B}}$, the apparent Doppler shift after correcting for gravitational effects caused by solar-system objects, and effects by the observer's displacement and motion relative to the solar-system barycenter. To first order this coincides with the classical concept of RV and for modest accuracies $\left(0.5 \mathrm{~km} \mathrm{~s}^{-1}\right.$, say), the new definition implies no change of existing procedures. However, the future use of $c z_{\mathrm{B}}$ will permit to exploit high-accuracy measurements for new classes of astrophysical tasks.

The definitions avoid any discussion of what the "true" RV would be, and a main point is that highly precise observations should be published (also) without the observer first trying to "calibrate" them against "standard" objects. Any subsequent interpretation of observed wavelength shifts in terms of the object's radial and transverse motion and other effects should be made separately. Since the uncertainties in any such modeling are much greater than those now reachable for the wavelength shifts themselves, precise observational data may become corrupted by applying model-dependent "corrections", thus precluding 
their use for possible more sophisticated analyses in the future. An example of such effects is the detection of small differences in the apparent RVs of different classes of photospheric lines due to convection (Allende Prieto et al. 2002).

\subsection{Radial Velocities of Galaxies}

\section{H. Quintana}

The three years covered by this report have seen the coming of age of a new generation of RV surveys of galaxies. These are characterized by one or two orders of magnitude increase in the number of redshifts obtained over previous ones, reaching over $10^{5}$ galaxies measured. The two most emblematic such massive surveys, now fully underway, are the Sloan Digital Sky Survey (SDSS) and the 2dF Galaxy Redshift Survey (2dFGRS). At present the SDSS has reached a number of new galaxy redshifts of order $2.5 \cdot 10^{5}\left(4 \cdot 10^{5}\right.$ including all objects), being at about $1 / 3$ of its completion target, and the 2dFGRS has reached $2.2 \cdot 10^{5}$, close to its target.

New research fields are being opened with these and future extensive surveys. Now it will be possible to quantitatively study hundreds of thousands of galaxies and to obtain high precision statistical results, from those datasets or from comparison with similar databases at other wavelengths. For the first time, optical data are providing strong constraints on cosmologies. Gone are the days of surveys done by a couple of astronomers and a few telescope nights of observations. Modern surveys require dedicated telescopes and/or spectrographs, years of continuous observations, teams of astronomers (often located in several countries) and substantial research funds.

Table 1. RV surveys of galaxies ( $N-$ target number of redshifts)

\begin{tabular}{lll}
\hline Survey & $N, 10^{3}$ & Reference, http:// \\
\hline SDSS & 750 & www.astro.princeton.edu/PBOOK/welcome.html \\
2dFGRS & 250 & www.mso.anu.edu.au/2dFGRS/Public/Survey \\
2MASS RS & 150 & $\begin{array}{l}\text { pegasus.phast.umass.edu } \\
\text { wdf }\end{array}$ \\
DEEP & 1.1 & $\begin{array}{l}\text { deep.ucolick.org } \\
\text { www.archive.noao.edu/ndwfs }\end{array}$ \\
NOAO deep-wide & & ww.arcoless/6dF \\
PSCz & 15 & www-astro.physics.ox.ac.uk/wjs/pscz.html \\
LCRS & 26.418 & manaslu.astro.utoronto.ca/lin/lcrs.html \\
Durham/UKST & 2.5 & star-www.dur.ac.uk/fhoyle/ukst.html \\
\hline \hline
\end{tabular}

Other important surveys, though less massive, have been completed or are underway (Table 1). Typically they are producing thousands of galaxy redshifts. As is now customary, all these projects have Web pages, describing the survey parameters and characteristics, sometimes catalogues and/or data are available on-line, complete documentation, including all the papers dealing with the subject and other relevant information for the interested users, is also available. For this reason here we only point the reader to these sources. Among these surveys are: the all-sky NIR selected 2MASS Redshift Survey, the NIR selected 6dF Survey carried out with FLAIR and related to the above, the DEEP - Deep Extragalactic Evolutionary Probe done with Keck and HST, the starting NOAO deep-wide survey for objects at $z>1$, and several surveys now completed, such as the PSCz Redshift Survey, providing redshifts of IRAS galaxies, the LCRS - Las Campanas Redshift Survey, the CNOC and ENACS cluster surveys, the CFRS, the Durham/UKST survey. We should also mention the Shapley supercluster survey that now reached some 6500 galaxy redshifts (Quintana et al. 2000), the Southern Abell cluster survey (Muriel et al., in press) with over 6000 velocities in 40 clusters. Furthermore, there are several galaxy redshift efforts in the HDF-S and other pencil beam imaging surveys. In the next few years to come we will see the emergence of large surveys done with multiobject (multislit and fibers) spectrographs at $8 \mathrm{~m}$-class telescopes, e.g. with VIMOS at VLT and with GMOS at Gemini. 


\subsection{The Milky Way}

\section{B. Nordström}

The Milky Way is the galaxy for which the most detailed dynamical information can be obtained, from distances, proper motions, and radial velocities of well-defined populations of stars. Because the properties of the Galaxy itself rather than its individual constituents are the subject of study, new surveys and catalogues of many galactic stars are the key resource in Galactic research.

The main such large new radial-velocity studies in the triennium are listed below, even though the main motivation for some of the surveys have been the study of certain types of objects such as extrasolar planet hosts or pulsating stars.

Galactic centre: Near the centre of the Milky Way, Ghez et al. (2000) have measured accelerations for several stars and found strong evidence for a supermassive black hole.

The thin and thick disks: A sample of 39 photometrically selected, very red $\mathrm{M}$ dwarfs was studied by Reid et al. (2002) from Keck/HIRES spectra. The velocity dispersions suggest that this newly-discovered ultra-cool population of $\mathrm{M}$ dwarfs is young.

Gilmore et al. (2002) redetermined the rotation of the thick disk from radial velocities of some $2000 \mathrm{~F} / \mathrm{G}$ stars obtained with the $2 \mathrm{dF}$ multi-fibre instrument. They find a much slower net rotation $\left(100 \mathrm{~km} \mathrm{~s}^{-1}\right)$ than expected and conclude that a single major merger event took place in the Galaxy some 10-12 Gyr ago.

Space motions for 39 metal-poor giant stars observed by Beers et al. (2002) indicate that the metal-weak thick disk extends to metallicities below $[\mathrm{Fe} / \mathrm{H}]=-1.6$.

Population II stars: First radial velocities for a sample of 114 halo stars, including 5 probable binaries, were published by Sperauskas \& Bartkevičius (2002). A large RV survey of halo stars conducted at CfA produced many spectroscopic orbits (Latham et al. 2002; Goldberg et al. 2002).

General: As part of a major effort to search for small-scale velocity variables and potential planet hosts, Nidever et al. (2002) studied radial velocities for 889 FGKM stars. With almost $90 \%$ of the stars having radial velocity variations of less than $200 \mathrm{~m} \mathrm{~s}^{-1}$, it is suggested that they might form the basis for a future set of standard stars.

A catalogue of mean radial velocities for 20574 stars was published by Barbier-Brossat and Figon (2000) and available from the CDS via anonymous $\mathrm{ftp}$ to cdsarc.u-strasbg.fr (130.79.128.5). The bibliographic catalogue of stellar radial velocities 1991-1994 (Malaroda et al. 2000) contains 13359 entries for stars in the Milky Way and the Magellanic Clouds. This catalogue is also available from the CDS.

\subsection{Star Clusters}

\section{N. Morrell}

Progress in this field is related to high-resolution spectroscopy at large telescopes which permits to measure RVs of faint stars with sufficient precision. Multi-object fiber-fed spectrographs are particularly well suited for cluster studies, reaching many targets simultaneously in reasonable time. Observations of very cool or obscured objects became possible through IR spectroscopy.

Open clusters. Substantial progress has been achieved on RVs of very young stellar clusters and star-forming regions. Modern techniques permit to reach pre-main sequence low-mass stars; the lithium $6708 \AA$ absorption is often used as a diagnostic of youth and cluster membership. The clusters studied are IC 4996, NGC 2264, $\lambda$ Ori region (Dolan \& Mathieu 1999), $\sigma$ Ori cluster (Zapatero Osorio et al. 2002), and M35.

Significant new data on RVs in intermediate-age open clusters have been obtained. They are used for membership, binarity, rotation studies. In the nearest clusters like Hyades and Pleiades, M-type dwarfs have been reached (Terndrup et al. 2000; Reid \& Mahoney 2000). Stars of higher masses have been observed in these nearby clusters as well, including 
Praesepe and Coma Berenices. For nearby clusters, it is possible to deduce RVs from proper motions; such astrometric radial velocities were derived by Madsen et al. (2002) and in the previous works of Lund Observatory group.

RVs were measured for members of more distant open clusters IC 2391, IC 2391, IC 2602, $\alpha$ Per, IC 4651, NGC 2451A, NGC 2516, NGC 3114, NGC 3532, NGC 6231, NGC 6811, NGC 2324, NGC 2818, NGC 3960, NGC 6259, and an old cluster NGC 2355. In LMC clusters SL 353 and SL 349, RVs of 22 individual stars were measured by Dieball et al. (2000); other LMC clusters studied are 30 Dor (Bosch et al. 2001) and R136 (Massey et al. 2002).

Moderate resolution near-IR spectroscopy (using CFHT, ESO and Keck facilities) yielded the first RV determinations for individual stars in the Galactic center open cluster (cf. Gezari et al. 2002 for the latest reference); comparisons with proper motions are consistent with a spherical star cluster around Sgr A.

A bibliographic database for stars in open clusters from which most published data can be retrieved is maintained by J.-C. Mermilliod (http://obswww.unige.ch/webda/).

Globular clusters. Measurements of RVs in globular clusters continue to be a fruitful field. For the most distant and reddened clusters, large telecopes are needed, like HIRES on the Keck I (Côté 1999). To overcome crowding in the central region of M15, Gebhardt et al. (2000) used adaptive optics at CFHT with Fabry-Perot. Other clusters studied include NGC 6528, Terzan 1, M3, M13, M15, M92, 47 Tuc, NGC 362, and the Pyxis globular cluster, believed to be an LMC cluster captured by the Milky Way.

\subsection{Spectroscopic Binaries}

\section{T. Mazeh}

The systematic RV search for spectroscopic binaries (SBs) within a large complete sample of more than 1400 high-proper-motion stars has been finally completed (Latham et al. 2002; Goldberg et al. 2002), yielding 171 single-lined binaries (SB1) and 34 doublelined ones (SB2). This rich sample of binaries increased dramatically our knowledge of the characteristics of the SB population with K-type primaries. The Geneva group extended the Duquennoy and Mayor G-type study to $\mathrm{K}$ stars (Mayor et al. 2001). In the meantime, the Geneva and the CfA teams (together with Tel Aviv research group) are monitoring a large sample of $>3000$ nearby $G$ stars. Although this effort did not yield any new publication in the last three years, this survey, when completed, will increase our information about SB with G-type primaries.

A pioneering work is done at the Geneva Observatory to determine the spectroscopic orbits of early-type stars with Elodie and Coralie, their high-precision high-resolution speedometers. They even succeeded to find an orbit of a $\delta$ Scuti A star, whose orbital variability was masked by the stellar pulsation (Carrier et al. 2002). This work has the potential of studying barely explored domains of the parameter space of SBs.

In the last three years, dedicated observations to detect secondary spectra and derive their RVs in known SB1s, thus turning them into SB2s, started to yield some interesting results. It has been shown that observations in the infrared could detect faint secondaries, especially if the binary is in its PMS phase (Prato et al. 2002).

We have seen recently many studies that combine RV orbits with astrometric/interferometric/photometric data that produce new results on stellar masses. Apart from a classical combination of spectroscopy and photometry for eclipsing SB2s, two approaches are used:

1. Derivation of the secondary masses (or at least upper limits for them) of SB1s, by combining astrometric data from the Hipparcos satellite with RV measurements, when the primary masses are estimated from their spectra (cf. Pourbaix \& Arenou 2001). These works corroborated the suggestion that the populations of planets and stellar secondaries are separated by their masses with the "brown dwarf desert". More results are expected from the next generation of astrometric satellites. 
2. Derivation of the masses of the two components of SB2s by combining RVs with astrometric/interferometric data, specially for PMS binaries (Steffen et al. 2001). A new $\mathrm{M}-\mathrm{L}$ relation at the bottom of the main-sequence is derived (e.g. Delfosse et al. 2000), leading to an interesting confrontation with stellar models.

\subsection{Stellar Oscillations and Other Pulsating Stars}

\section{Dravins \& L. Szabados}

In the last 3 years, the p-mode oscillations in solar-type stars other than Sun have finally been detected, opening a new field of astroseismology that provides direct information on stellar interior structure. After attempts to use various photometric and spectroscopic techniques, a breakthrough has occurred with precise RV measurements clearly detecting many individual acoustic modes in several ordinary stars. The observations, requiring precisions on the $\mathrm{m} \mathrm{s}^{-1}$ level, were made with spectrometers developed primarily for exoplanet searches.

Possibly, the first such significant detection was for Procyon (F5 IV-V) (Martic et al. 1999), although the power spectrum was found to be less regular than solar. The p-mode oscillation spectrum in $\beta$ Hyi (G2 IV) was detected with different instruments by Bedding et al. (2001) and Carrier et al. (2001). Typical frequencies are around $1 \mathrm{mHz}$ (periods $\approx 15$ min, three times longer than in the Sun), reflect the larger size and lower surface gravity in this subgiant. Analogous oscillations in the solar near-twin $\alpha$ Cen A (G2 V) were seen by Bouchy \& Carrier (2002).

The precise determination of oscillation frequencies requires long observation periods (weeks) and high instrumental stability, especially challenging for the long oscillation periods in giant stars. For $\xi$ Hya (G7 III), a month-long observing campaign was needed to map out oscillation periods of typically 3 hours (ESO press release, 10/02 2002).

Extensive work on other, "classical" types of pulsating variables done in the past triennium is briefly covered below.

Cepheids. The most extensive observing effort on RVs of cepheids (about $1500 \mathrm{RVs}$ ) during the recent 3 years was that of the Moscow team (N.A. Gorynya et al.). These data have been analyzed for detection of new binaries and for properties of the pulsators, with the use of the Fourier-decomposition techniques (P. Moskalik, Warsaw). Pont et al. (2001) carried out morphological study of RV curves of outer-disk Cepheids based on new data; no effect of metal abundance on RV curves was found.

Bersier (2002) revealed or confirmed the spectroscopic binary nature of U Car, XX Car, T Cru, WZ Sgr, and SY Nor, while Evans (2000) determined a 5-yr orbit for V1334 Cyg form 30 yr of data. The precise RV measurements of Polaris - a Cepheid also belonging to an SB system - show additional intrinsic variation superimposed on the pulsational and orbital effects (Hatzes \& Cochran, 2000) whose origin has not been clarified yet. It is also important that the pulsational amplitude of Polaris stopped decreasing.

Gamma Doradus type variables. From the newly defined type of pulsating variables, HR 8799 was studied most thoroughly (Zerbi et al. 1999): the multi-site photometric campaign was supplemented with a set of RV data.

Mira type and other long-period variables. Alvarez et al. (2001) performed envelope tomography for long-period variable stars. Scholz \& Wood (2000) calculated model atmospheres for Miras and concluded that they pulsate in the first overtone mode.

\subsection{Extrasolar Planets}

\section{N.C. Santos \& S. Udry}

The period covered by this report has seen tremendous developments in the exoplanet domain. Mostly resulting from the increase in the baseline of the RV data and the enlargement of the survey samples, the planet list has grown from merely 20 planets to now more than 100 . The variety of planetary characteristics observed with the first candidates 
is developing further. We have: longer-period candidates, a large planetary mass range from sub-saturnian (Marcy et al. 2000; Pepe et al. 2002) up to masses above $10 \mathrm{M}_{\mathrm{Jup}}$, and candidates with very high eccentricities up to $e=0.92$ (Naef et al. 2001). With longer period objects becoming detectable, some planets are now found to resemble our Solar System giant planets. The number of multi-planetary systems reached about 11 today. Several of them are found in resonant configurations like e.g. period ratios of 2:1. In such systems, planet-planet interactions bring further information on the orbital inclinations. Planets orbiting components of wide and spectroscopic binaries have been also detected.

This strong increase in the number of planetary candidates has boosted various new studies that are opening a complete new window into the mechanisms of planetary formation and evolution. The "magic" number of 100 , more than having a philosophical meaning, means that we are now able to do significant statistical analyses of the exoplanet properties. These have permitted to confirm former suggested trends, as well as to unveil new ones. The distribution of the planetary mass is found to be steeply rising towards the low mass regime, even when we correct for the orbital inclination effects (Jorissen et al. 2001, Zucker $\&$ Mazeh 2001). The maximum mass of a planet is estimated to be around $10 \mathrm{M}_{\mathrm{Jup}}$, a limit that does not have anything to do with the deuterium burning limit of $13 \mathrm{M}_{\mathrm{Jup}}$.

The study of the orbital period distribution has shown the importance of considering migration processes to explain the observed current configuration of the planetary systems (piling-up of planets with periods around 3 days, rise of the number of massive planets with distance). Also a paucity of high mass companions orbiting in short period trajectories has been pointed out (Zucker \& Mazeh 2002; Udry et al. 2002). These results bring strong constraints for the migration mechanisms.

Further information comes from the study of the planet hosts themselves. They are found to be particularly metal rich when compared to average field dwarfs (Gonzalez et al. 2001; Santos et al. 2001). This tendency, of probable primordial origin, indicates that planet formation is strongly dependent of the metallicity of the cloud of gas and dust that gave birth to the star and planetary system. Some possible evidences of the infall of planetary material (or even planets) were however discovered in HD 82943 in the form of an anomalous lithium isotopic ratio (Israelian et al. 2001).

The exoplanet research was not restricted to the RVs. The most prominent result is the detection of the transit of a giant planet in front of the star HD 209458 (Charbonneau et al. 2000; Henry et al. 2000). This detection not only represents an independent confirmation of the nature of the discovered body, but also provides planetary physical properties, like its mass, radius, or mean density. Subsequent studies of this system have permitted to show that the planet around HD 209458 is orbiting in the plane of the stellar equator (Queloz et al. 2000). Furthermore, recent spectroscopic studies have permitted to unveil some details about the composition of the atmosphere of the planet itself (Charbonneau et al. 2002). Although RV methods provide for the moment the more efficient way to search for exoplanets, wide-field photometric planetary transit surveys may open a new era, revealing thousands of transits in a near future.

\section{Working Groups}

\subsection{Standard Radial-Velocity Stars}

Members: S. Udry (chair), R. Stefanik, and F. Fekel. The tedious work on RV standards was continued with no new results as yet.

\subsection{Bibliography of Stellar Radial Velocities}

Members: H. Levato (chair) and S. Malaroda, with technical support from M. Grosso, S. Galliani, and A. Rodas. The WG has updated the Bibliographic Catalogue of Radial Velocities during this period at a rate of a new update each semester. At the time of this writing the update up to December 31,2001 has been published and the version 2002.5 will 
be available before the end of October 2002 at the WEB site http://www.casleo.gov.ar and at the CDS. The last published version has almost 45000 entries, doubling this number over the past 5 years.

\title{
3.3. Ninth Catalogue of the Orbital Elements of Spectroscopic Binary Stars
}

Members: A. Tokovinin (chair), A. Batten, F. Fekel, W. Hartkopf, D. Latham, H. Levato, B. Mason, N. Morell, D. Pourbaix, S. Udry. This WG was created in 2000 to compile the 9th catalogue of orbits of spectroscopic binaries (SB9), superseding the SB8 of Batten et al. (1989). SB9 exists in electronic format only; unlike SB8, it contains also individual radial velocities (at least for new orbits where they are available) and is continuously updatable.

The SB9 web site http://sb9.astro.ulb.ac.be was officially released in summer 2001 . It was implemented by D. Pourbaix and provides convenient interface with object search capabilities, output of all data and graphs. Since November 13, 2001, there were 1931 queries to SB9 from 154 different IP addresses, showing a high degree of community interest. SB9 is linked to the web pages of IAU Commissions 30 and 26 as well as to the Besançon database on binary stars, http://bdb.obs-besancon.fr.

As of October 1, 2002, SB9 contained 1707 systems (1469 in SB8) and 1756 orbits (1469 in SB8). A total of 70 papers were added, but most of them not by the WG members, as was planned initially. We still have a list of 304 identified papers whose orbits need to be entered.

\author{
A.A. Tokovinin \\ President of the Commission
}

\section{References}

Allende Prieto C., Asplund M., García López R.J., \& Lambert D.L. 2002, ApJ, 567, 544

Alvarez R., Jorissen A., Plez B. et al. 2001, A\&A, 379, 305

Barbier-Brossat M. \& Figon P. 2000, A\&AS, 142, 217

Batten A.H., Fletcher J.M., MacCarthy D.G. 1989, Publ. DAO, 17

Bedding T.R., Butler R.P., Kjeldsen H. et al. 2001, ApJ, 549, L105

Beers T.C., Drilling J.S., Rossi S. et al. 2002, AJ, 124, 931

Bersier D. 2002, ApJS, 140, 465

Bosch G., Selman F., Melnick J., Terlevich R. 2001, A\&A, 380, 137

Bouchy F. \& Carrier F. 2002, A\&A, 390, 205

Carrier F., Bouchy F., Kienzle F. et al. 2001, A\&A, 378, 142

Carrier F., Debernardi Y., Udry S. et al. 2002, A\&A, 390, 1027

Charbonneau D., Brown T., Noyes R., Gilliland R. 2002, ApJ, 568, 377

Charbonneau D., Brown T., Latham D., Mayor M. 2000, ApJ, 529, L45

Côté P. 1999, AJ, 118, 406

Delfosse X., Forveille T., Ségransan D. et al. 2000, A\&A, 364, 217

Dieball A., Grebel E. K., Theis C. 2000, A\&A, 358, 144

Dolan C.J. \& Mathieu R. 1999 AJ, 118, 2409

Dravins D., Lindegren L., \& Madsen S. 1999, A\&A, 348, 1040

Evans N.R. 2000, AJ, 119, 3050

Gebhardt K., Pryor C., O'Connell R. D. et al. 2000, AJ, 119, 1268

Gezari S., Ghez A. M., Becklin E. E. et al. 2002, ApJ, 576, 790

Ghez A.M., Morris M., Becklin E.E. et al. 2000, Nature, 407, 349

Gilmore G., Wyse R.F.G., Norris J.E. 2002, ApJ, 574, L39 
Goldberg D., Mazeh T., Latham D. W. et al. 2002, AJ, 124, 1132

Gonzalez G., Laws C., Tyagi S., Reddy B. 2001, AJ, 121, 432

Hatzes A.P. \& Cochran W.D. 2000, AJ, 120, 979

Henry G.W., Marcy G.W., Butler R.P., \& Vogt S.S. 2000, ApJ, 529, L41

Israelian G., Santos N.C., Mayor M., Rebolo R. 2001, Nature, 411, 163

Jorissen A., Mayor M., Udry S. 2001, A\&A, 379, 992

Latham D. W., Stefanik R. P., Torres, G. et al. 2002, AJ, 124, 1144

Madsen S., Dravins D. \& Lindegren L. 2002, A\&A, 381, 446

Malaroda S., Levato H., Morrell N. et al. 2000, A\&AS, 144, 1

Marcy G., Butler P., Vogt S. 2000, ApJ, 536, L43

Martic M., Schmitt J., Lebrun J.-C. et al. 1999, A\&A, 351, 993

Massey P., Penny L. R., Vukovich J. 2002, ApJ, 565, 982

Mayor M., Udry S., Halbwachs J.-L., \& Arenou F. 2001, Proc. IAU Symp. 200 The formation of binary stars, ed. H. Zinnecker \& R.D. Mathieu, 45

Naef D., Latham D., Mayor M. et al. 2001, A\&A, 375, L27

Nidever D.L., Marcy G., Butler P.R. et al. 2002, ApJS, 141, 503

Pepe F., Mayor M., Galland F. et al. 2002, A\&A, 388, 632

Prato L., Simon M., Mazeh T. et al. 2002, ApJ, 569, 863

Pont F., Kienzle F., Gieren W., \& Fouqué P. 2001, A\&A, 376, 892

Pourbaix D. \& Arenou F. 2001, A\&A, 372, 935

Queloz D., Eggenberger A., Mayor M. et al. 2000, A\&A, 359, L13

Quintana H., Carrasco E. R., \& Reisenegger A. 2000, AJ, 120, 511

Reid I.N. \& Mahoney S. 2000, MNRAS, 316, 827

Rickman H. 2002, IAU Inf.Bull., 91, 50

Santos N.C., Israelian G., \& Mayor M. 2001, A\&A, 373, 1019

Scholz M. \& Wood P.R. 2000, A\&A, 362, 1065

Sperauskas J. \& Bartkevičius A. 2002, AN, 323, 139

Steffen A. T., Mathieu R. D., Lattanzi M. G. et al. 2001, AJ, 122, 997

Terndrup D.M., Stauffer J.R., Pinsonneault M.H. et al. 2000, AJ, 119, 1303

Udry S., Mayor M., Naef D. et al. 2002, A\&A, 390, 267

Zapatero Osorio M.R., Bèjar V. J. S., Martín E. L. et al. 2002, A\&A, 384, 937

Zerbi F. M., Rodriguez E., Garrido R. et al. 1999, MNRAS, 303, 275

Zucker S. \& Mazeh T. 2001, ApJ, 562, 1038

Zucker S. \& Mazeh T. 2002, ApJ, 568, L113 